\title{
Energy Efficient Transmission in MIMO Interference Channels with QoS Constraints
}

\author{
Yang Yang ${ }^{\dagger}$ and Marius Pesavento ${ }^{\ddagger}$ \\ ${ }^{\dagger}$ Intel Deutschland GmbH, Neubiberg 85579, Germany. \\ $\ddagger$ Technische Universität Darmstadt, Darmstadt 64283, Germany. \\ Emails: yang1.yang@intel.com, pesavento@nt.tu-darmstadt.de
}

\begin{abstract}
In this paper, we consider the energy efficiency maximization problem in MIMO interference channels where all users have a guaranteed minimum transmission rate. To solve this optimization problem with a nonconcave objective function and a nonconvex constraint set, we extend the recently developed successive pseudoconvex approximation framework and propose a novel iterative algorithm that has the following advantages: 1) fast convergence, as the structure of the original optimization problem is preserved as much as possible in the approximate problem solved in each iteration, 2) efficient implementation, as each approximate problem is natural for parallel computation and its solution has a closed-form expression, and 3) guaranteed convergence to a KarushKuhn-Tucker (KKT) point.
\end{abstract}

Index Terms-Interference Channel, MIMO, Nonconvex Optimization, Pseudoconvex optimization, QoS

\section{INTRODUCTION}

With the advent of $5 \mathrm{G}$ by 2020 , the number of connected devices is predicted to reach 50 millions with a targeted 10-fold increase of data rate. The increase in the data rate is expected to be achieved at the same or even a lower level of energy consumption. Therefore the so-called energy efficiency (EE) of the network is a key performance indicator that attracts extensive interest and it imposes stringent requirements on efficient transmission schemes enhancing EE.

In this paper, we study the EE maximization problem in MIMO interference channels (IC) where all users have a Quality-of-Service $(\mathrm{QoS})$ guarantee in terms of the minimum transmission rate. It is well known that sum rate maximization in MIMO IC is a NP-hard problem [1]. The EE maximization problem in MIMO IC is an even more challenging problem because the $\mathrm{EE}$ is a fractional function (with the consumed energy in the denominator) while the sum rate function in the numerator is a nonconcave function [2], and QoS constraints make the constraint set nonconvex.

In state-of-the-art studies, the EE maximization problem in interference-limited systems has received considerable attention, see $[2,3,4,5,6,7]$ and the references therein. The iterative algorithms proposed in $[2,3,7]$ have taken the QoS constraints into consideration, but they generally suffer from a high complexity because the problems solved in each iteration do not have structures for which efficient parallel implementations are known, and they are mostly solved by convex optimization solvers and this may incur latencies in the decision making process. The algorithm proposed in [5] is a variant of the well-known block coordinate descent algorithm and it relies on the uncoupling structure in the constraint set. Although it can be extended to a MIMO system, it cannot properly handle the QoS constraints, which not only make the constraint nonconvex but also couple the users' feasible transmit strategies. In [6], we proposed a parallel algorithm that is of a best-response type based on the unified successive pseudoconvex approximation (SPCA) framework to maximize the EE in the IC, where the notation of pseudoconvexity,

The work of Yang is supported by the framework of the Horizon 2020 project ONE5G (ICT-7-2016). a weaker form of convexity [8, 9], plays an essential role in the convergence proof. However, the framework is only applicable when the constraint set is convex, which is not the case in the problem under consideration due to nonconvexity of the QoS constraints.

In this paper, we extend the SPCA framework proposed in [6] to solve optimization problems with a nonconvex constraint set, and develop an iterative algorithm to maximize the EE in MIMO IC with QoS constraints. In each iteration, an approximate problem is solved, where the approximate set is a convex (inner) approximation of the original nonconvex constraint set, and the approximate function only needs to be pseudoconvex, a weak form of convexity. This weak assumption makes it possible to preserve as much structure available in the original EE function as possible, e.g., the partial concavity in the numerator function and the division operator. Besides this, the proposed approximate problem is natural for parallel computation, as the approximate problem can be decomposed into many independent subproblems that can be solved in parallel and each subproblem has a closed-form solution.

\section{Problem Model}

We consider the downlink of a MIMO multi-cell system where the BS in each cell is serving a single user on given frequency resource, and $\mathbf{H}_{k j}$ is the channel coefficient matrix from the BS $j$ to the user $k$. Assume the multi-user interference is treated as noise, the transmission rate of the $k$-th user is:

$$
r_{k}\left(\mathbf{Q}_{k}, \mathbf{Q}_{-k}\right)=\log \operatorname{det}\left(\mathbf{I}+\mathbf{R}_{k}\left(\mathbf{Q}_{-k}\right)^{-1} \mathbf{H}_{k k} \mathbf{Q}_{k} \mathbf{H}_{k k}^{H}\right),
$$

where $\mathbf{Q}_{k}$ is user $k$ 's transmit covariance matrix, $\mathbf{Q}_{-k}=\left(\mathbf{Q}_{j}\right)_{j \neq k}$ is a compact notation denoting the collection of all transmit covariance matrices except $\mathbf{Q}_{k}$, and $\mathbf{R}_{k}\left(\mathbf{Q}_{-k}\right) \triangleq \sigma_{k}^{2} \mathbf{I}+\sum_{j \neq k} \mathbf{H}_{k j} \mathbf{Q}_{j} \mathbf{H}_{k j}^{H}$ is covariance matrix of the noise (with variance $\sigma_{k}^{2}$ ) plus interference. The transmit covariance matrices $\mathbf{Q}=\left(\mathbf{Q}_{k}\right)_{k=1}^{K}$ are constrained to be in a feasible set $\mathcal{Q}$ defined as:

$$
\mathcal{Q} \triangleq\left\{\mathbf{Q}: \mathbf{Q}_{k} \succeq \mathbf{0}, \operatorname{tr}\left(\mathbf{Q}_{k}\right) \leq P_{k}, r_{k}(\mathbf{Q}) \geq R_{k}, \forall k\right\},
$$

where the constant $\underline{R}_{k}$ is the minimum guaranteed transmission rate achieved by the user $k$, and $r_{k}(\mathbf{Q}) \geq \underline{R}_{k}$ thus serves as a QoS constraint for user $k$.

The network EE optimization problem is thus to maximize the EE, defined as the ratio between the sum transmission rate and the total consumed power, over the constraint set $\mathcal{Q}$ :

$$
\underset{\mathbf{Q} \in \mathcal{Q}}{\operatorname{maximize}} f(\mathbf{Q}) \triangleq \frac{\sum_{k=1}^{K} r_{k}\left(\mathbf{Q}_{k}, \mathbf{Q}_{-k}\right)}{\sum_{k=1}^{K}\left(P_{0, k}+\rho_{k} \operatorname{tr}\left(\mathbf{Q}_{k}\right)\right)},
$$

where $P_{0, k}$ and $\rho_{k}$ are the constant scalars specifying the power consumption at the zero RF output power (i.e., $\mathbf{Q}_{k}=\mathbf{0}$ ) and the slope of the load dependent power consumption, respectively. The values of $P_{0, k}$ and $\rho_{k}$ depend on the types of the cell, e.g., macro 
cell, remote radio head, or micro cell [10, Table 8]. We assume that $\mathcal{Q}$ is nonempty and problem (3) has a solution.

\section{The Proposed Successive Pseudoconvex APPROXIMATION ALGORITHM}

To design an iterative algorithm for problem (3) that enjoys a low complexity but at the same time a fast convergence behavior, we need on the one hand to overcome the nonconvexity in both the objective function and the constraint set, and, on the other hand, to preserve the original problem's structure as much as possible. Towards this end, we extend the successive pseudoconvex approximation framework developed in [6] for minimizing a nonconvex function over a convex constraint set to solve problem (3) where both the objective function and the constraint set are nonconvex.

The proposed iterative algorithm consists of solving a sequence of successively refined approximate problems. In iteration $t$, the approximate problem defined around the point $\mathbf{Q}^{t}$ consists of maximizing an approximate function, denoted as $\tilde{f}\left(\mathbf{Q} ; \mathbf{Q}^{t}\right)$, over an (inner) approximate set, denoted as $\tilde{\mathcal{Q}}\left(\mathbf{Q}^{t}\right)$. Both $\tilde{f}\left(\mathbf{Q} ; \mathbf{Q}^{t}\right)$ and $\tilde{\mathcal{Q}}\left(\mathbf{Q}^{t}\right)$ should exhibit some form of convexity so that the approximate is much easier to solve than the original problem (3).

Approximate function. The numerator functions $\left\{r_{k}(\mathbf{Q})\right\}$ in (3) are not concave in $\mathbf{Q}$, and thus the objective function $f(\mathbf{Q})$ is not even pseudoconcave. Meanwhile, the function $r_{k}(\mathbf{Q})$ is concave in $\mathbf{Q}_{k}$, and exploiting this partial concavity may notably accelerate the convergence [11]. Therefore, we construct an approximate function for the variable $\mathbf{Q}_{k}$, denoted as $\tilde{r}_{k}\left(\mathbf{Q}_{k} ; \mathbf{Q}^{t}\right)$, by fixing the remaining variables $\mathbf{Q}_{-k}$ in $r_{k}\left(\mathbf{Q}_{k}, \mathbf{Q}_{-k}\right)$ and linearizing only the functions $\left\{r_{j}(\mathbf{Q})\right\}_{j \neq k}$ that are not concave in $\mathbf{Q}_{k}$ :

$$
\tilde{r}_{k}\left(\mathbf{Q}_{k} ; \mathbf{Q}^{t}\right) \triangleq r_{k}\left(\mathbf{Q}_{k}, \mathbf{Q}_{-k}^{t}\right)+\sum_{j \neq k}\left(\mathbf{Q}_{k}-\mathbf{Q}_{k}^{t}\right) \bullet \nabla_{\mathbf{Q}_{k}^{*}} r_{j}\left(\mathbf{Q}^{t}\right),
$$

where $\mathbf{A} \bullet \mathbf{B}=\Re\left(\operatorname{tr}\left(\mathbf{A}^{H} \mathbf{B}\right)\right)$. Since $\tilde{r}_{k}\left(\mathbf{Q}_{k} ; \mathbf{Q}^{t}\right)$ is concave in $\mathbf{Q}_{k}$, $\sum_{k=1}^{K} \tilde{r}_{k}\left(\mathbf{Q}_{k} ; \mathbf{Q}^{t}\right)$ is concave in $\mathbf{Q}$. This paves the way to define an approximate function $\tilde{f}\left(\mathbf{Q} ; \mathbf{Q}^{t}\right)$ of the following form:

$$
\tilde{f}\left(\mathbf{Q} ; \mathbf{Q}^{t}\right) \triangleq \frac{\sum_{k=1}^{K} \tilde{r}_{k}\left(\mathbf{Q}_{k} ; \mathbf{Q}^{t}\right)}{\sum_{k=1}^{K}\left(P_{0, k}+\rho_{k} \operatorname{tr}\left(\mathbf{Q}_{k}\right)\right)},
$$

and it has the following important properties:

- The approximate function $\tilde{f}\left(\mathbf{Q} ; \mathbf{Q}^{t}\right)$ is still nonconcave, but it is the fractional function of a concave function and a linear function, which is thus pseudoconcave [6].

- The gradient of $\tilde{f}\left(\mathbf{Q} ; \mathbf{Q}^{t}\right)$ and that of $f(\mathbf{Q})$ are identical at the point $\mathbf{Q}^{t}$ around which $\tilde{f}\left(\mathbf{Q} ; \mathbf{Q}^{t}\right)$ is defined:

$$
\nabla_{\mathbf{Q}^{\star}} \tilde{f}\left(\mathbf{Q}^{t} ; \mathbf{Q}^{t}\right)=\nabla f\left(\mathbf{Q}^{t}\right) .
$$

These properties correspond to the convergence conditions of the SPCA framework in [6] and they play an essential role in establishing the convergence of the proposed algorithm.

Approximate set: The function $r_{k}(\mathbf{Q})$ defined in (1) is nonconcave and it is the difference of two concave functions:

$$
r_{k}(\mathbf{Q})=r_{k}^{+}(\mathbf{Q})-r_{k}^{-}(\mathbf{Q}),
$$

where $r_{k}^{+}(\mathbf{Q}) \triangleq \log \operatorname{det}\left(\sigma_{k}^{2} \mathbf{I}+\sum_{j=1}^{K} \mathbf{H}_{k j} \mathbf{Q}_{j} \mathbf{H}_{k j}^{H}\right)$ and $r_{k}^{-}(\mathbf{Q}) \triangleq$ $\log \operatorname{det}\left(\sigma_{k}^{2} \mathbf{I}+\sum_{j \neq k} \mathbf{H}_{k j} \mathbf{Q}_{j} \mathbf{H}_{k j}^{H}\right)$. It follows from the definition of concave functions that $r_{k}^{-}(\mathbf{Q})$ is upper bounded by its first order approximation:

$$
\begin{aligned}
r_{k}^{-}(\mathbf{Q}) & \leq r_{k}^{-}\left(\mathbf{Q}^{t}\right)+\sum_{j \neq k}\left(\mathbf{Q}_{j}-\mathbf{Q}_{j}^{t}\right) \bullet \nabla_{\mathbf{Q}_{j}^{*}} r_{k}^{-}\left(\mathbf{Q}^{t}\right) \\
& \triangleq \bar{r}_{k}^{-}\left(\mathbf{Q} ; \mathbf{Q}^{t}\right)
\end{aligned}
$$

A differentiable function $h(\mathbf{x})$ is pseudoconcave if $h(\mathbf{x})>h(\mathbf{y})$ implies $(\mathbf{x}-\mathbf{y})^{T} \nabla h(\mathbf{y})>0$ for any two feasible points $\mathbf{x}$ and $\mathbf{y}$. where equality holds at $\mathbf{Q}=\mathbf{Q}^{t}$. Thus $r_{k}^{+}(\mathbf{Q})-\bar{r}_{k}^{-}\left(\mathbf{Q} ; \mathbf{Q}^{t}\right)$ is a global lower bound of $r_{k}(\mathbf{Q})$ :

$$
\begin{aligned}
r_{k}(\mathbf{Q}) & =r_{k}^{+}(\mathbf{Q})-r_{k}^{-}(\mathbf{Q}) \\
& \geq r_{k}^{+}(\mathbf{Q})-\bar{r}_{k}^{-}\left(\mathbf{Q} ; \mathbf{Q}^{t}\right) \triangleq \underline{r}_{k}\left(\mathbf{Q} ; \mathbf{Q}^{t}\right),
\end{aligned}
$$

where equality holds at $\mathbf{Q}=\mathbf{Q}^{t}$.

We define the (inner) approximate constraint set $\tilde{\mathcal{Q}}\left(\mathbf{Q}^{t}\right)$ by replacing the nonconcave functions $r_{k}(\mathbf{Q})$ with its lower bound $\underline{r}_{k}\left(\mathbf{Q} ; \mathbf{Q}^{t}\right)$ :

$$
\tilde{\mathcal{Q}}\left(\mathbf{Q}^{t}\right) \triangleq\left\{\begin{array}{ll}
\mathbf{Q}: \quad & \mathbf{Q}_{k} \succeq \mathbf{0}, \operatorname{tr}\left(\mathbf{Q}_{k}\right) \leq P_{k}, \\
& r_{k}^{+}(\mathbf{Q})-\bar{r}_{k}^{-}\left(\mathbf{Q}_{-k} ; \mathbf{Q}^{t}\right) \geq R_{k}, \forall k
\end{array}\right\} .
$$

Since $r_{k}^{+}(\mathbf{Q})-\bar{r}_{k}\left(\mathbf{Q} ; \mathbf{Q}^{t}\right)$ is concave, the set $\tilde{\mathcal{Q}}\left(\mathbf{Q}^{t}\right)$ is convex.

Approximate problem. In iteration $t$, the approximate problem defined at the point $\mathbf{Q}^{t}$ is:

$$
\underset{\mathbf{Q} \in \tilde{\mathcal{Q}}\left(\mathbf{Q}^{t}\right)}{\operatorname{maximize}} \quad \tilde{f}\left(\mathbf{Q} ; \mathbf{Q}^{t}\right)
$$

where the approximate function $\tilde{f}\left(\mathbf{Q} ; \mathbf{Q}^{t}\right)$ and the approximate set is defined in (4) and (7), respectively. We denote as $\mathbb{B} \mathbf{Q}^{t}$ a solution of problem (8) and $\mathcal{S}\left(\mathbf{Q}^{t}\right)$ the solution set:

$$
\mathcal{S}\left(\mathbf{Q}^{t}\right) \triangleq\left\{\mathbb{B} \mathbf{Q}^{t}: \mathbb{B} \mathbf{Q}^{t}=\underset{\mathbf{Q} \in \tilde{\mathcal{Q}}\left(\mathbf{Q}^{t}\right)}{\arg \max } \tilde{f}\left(\mathbf{Q} ; \mathbf{Q}^{t}\right)\right\} .
$$

It turns out that $\mathbb{B} \mathbf{Q}^{t}-\mathbf{Q}^{t}$ is an ascent direction of the original objective function $f(\mathbf{Q})$ at $\mathbf{Q}=\mathbf{Q}^{t}$, unless $\mathbf{Q}^{t}$ is already a KKT point of problem (3):

Proposition 1 (KKT point and ascent direction). A point $\mathbf{X}$ is a $K K T$ point of (3) if and only if $\mathbf{X} \in \mathcal{S}(\mathbf{X})$. If $\mathbf{X}$ is not a stationary point of (3), then $\mathbb{B} \mathbf{X}-\mathbf{X}$ is an ascent direction of $r(\mathbf{Q})$ in the sense that

$$
(\mathbb{B} \mathbf{X}-\mathbf{X}) \bullet \nabla f(\mathbf{X})>0 .
$$

Proof: Suppose $\mathbf{X} \in \mathcal{S}(\mathbf{X})$. By definition $\mathbf{X}$ solves the following optimization problem:

$$
\begin{array}{ll}
\underset{\mathbf{Q}}{\operatorname{maximize}} & \tilde{f}(\mathbf{Q} ; \mathbf{X}) \\
\text { subject to } & \mathbf{Q}_{k} \succeq \mathbf{0}, \operatorname{tr}\left(\mathbf{Q}_{k}\right) \leq P_{k}, \underline{r}_{k}(\mathbf{Q} ; \mathbf{X}) \geq R_{k}, \forall k,
\end{array}
$$

where $\underline{r}_{k}(\mathbf{Q} ; \mathbf{X})$ is defined in (6). Since $\mathbf{X}$ is a regular point [12], there exists $\left(\boldsymbol{\Pi}_{k}, \mu_{k}, \lambda_{k}\right)_{k=1}^{K}$ such that $\mathbf{X}$ and $\left(\boldsymbol{\Pi}_{k}, \mu_{k}, \lambda_{k}\right)_{k=1}^{K}$ together satisfy the KKT conditions [13, Prop. 4.3.1]:

$$
\begin{gathered}
\nabla \tilde{f}(\mathbf{X} ; \mathbf{X})+\mathbf{\Pi}_{k}-\sum_{k=1}^{K} \mu_{k} \mathbf{I}+\sum_{k=1}^{K} \lambda_{k} \nabla \underline{r}_{k}(\mathbf{X} ; \mathbf{X})=\mathbf{0}, \\
\boldsymbol{\Pi}_{k} \succeq \mathbf{0}, \mathbf{Q}_{k} \succeq \mathbf{0}, \boldsymbol{\Pi}_{k} \bullet \mathbf{Q}_{k}=0, k=1, \ldots, K, \\
\mu_{k} \geq 0, \operatorname{tr}\left(\mathbf{X}_{k}\right) \leq P_{k}, \mu_{k} \cdot \operatorname{tr}\left(\mathbf{X}_{k}\right)=0, k=1, \ldots, K, \\
\lambda_{k} \geq 0, \underline{r}_{k}(\mathbf{X} ; \mathbf{X}) \geq R_{k}, \lambda_{k} \cdot \underline{r}_{k}(\mathbf{X} ; \mathbf{X})=0, k=1, \ldots, K .
\end{gathered}
$$

Since $\nabla \tilde{f}(\mathbf{X} ; \mathbf{X})=\nabla f(\mathbf{X})$ and $\nabla \underline{r}_{k}(\mathbf{X} ; \mathbf{X})=\nabla r_{k}(\mathbf{X})$ according to (5) and (6), respectively, (11) can be rewritten as

$$
\nabla f(\mathbf{X})+\mathbf{\Pi}_{k}-\sum_{k=1}^{K} \mu_{k} \mathbf{I}+\sum_{k=1}^{K} \lambda_{k} \nabla r_{k}(\mathbf{X})=\mathbf{0} .
$$

Therefore $\mathbf{X}$ satisfies the KKT conditions of problem (3).

If, reversely, there exist $\left(\boldsymbol{\Pi}_{k}, \mu_{k}, \lambda_{k}\right)_{k=1}^{K}$ and $\mathbf{X}$ satisfying the KKT conditions of (3), it is straightforward to see that it also satisfies the KKT conditions of (10). Since the objective function in (10) is pseudoconcave and the constraint set $\overline{\mathcal{Q}}(\mathbf{X})$ is convex, it follows from [8, Th. 10.1.1] that $\mathbf{X}$ is an optimal solution of (10), i.e., $\mathbf{X} \in \mathcal{S}(\mathbf{X})$.

If $\mathbf{X} \notin \mathcal{S}(\mathbf{X})$, then $\tilde{f}(\mathbb{B} \mathbf{X} ; \mathbf{X})<\tilde{f}(\mathbf{X} ; \mathbf{X})$. Since $\tilde{f}(\mathbf{Q} ; \mathbf{X})$ is pseudoconcave, $0<(\mathbb{B} \mathbf{X}-\mathbf{X}) \bullet \nabla \tilde{f}(\mathbf{X} ; \mathbf{X})=(\mathbb{B} \mathbf{X}-\mathbf{X}) \bullet \nabla f(\mathbf{X})$ and $\mathbb{B} \mathbf{X}-\mathbf{X}$ is thus an ascent direction of $f(\mathbf{Q})$ at $\mathbf{Q}=\mathbf{X}$.

Since $\mathbb{B} \mathbf{Q}^{t}-\mathbf{Q}^{t}$ is an ascent direction of $f(\mathbf{Q})$ at $\mathbf{Q}=\mathbf{Q}^{t}$ according to Proposition 1, there exists a scalar $\gamma^{t} \in(0,1]$ such 
that $f\left(\mathbf{Q}^{t}+\gamma^{t}\left(\mathbb{B} \mathbf{Q}^{t}-\mathbf{Q}^{t}\right)\right)>f\left(\mathbf{Q}^{t}\right)$. In practice, the stepsize $\gamma^{t}$ is usually obtained by the so-called successive line search. That is, given two scalars $0<\alpha<1$ and $0<\beta<1, \gamma^{t}$ is set to be $\gamma^{t}=\beta^{m_{t}}$, where $m_{t}$ is the smallest nonnegative integer $m$ satisfying the following inequality:

$$
f\left(\mathbf{Q}^{t}+\beta^{m}\left(\mathbb{B} \mathbf{Q}^{t}-\mathbf{Q}^{t}\right)\right) \geq f\left(\mathbf{Q}^{t}\right)+\alpha \beta^{m} \nabla f\left(\mathbf{Q}^{t}\right) \bullet\left(\mathbb{B} \mathbf{Q}^{t}-\mathbf{Q}^{t}\right) .
$$

Note that the successive line search is carried out over the original objective function $f(\mathbf{Q})$ defined in (3).

After the stepsize $\gamma^{t}$ is found, the variable $\mathbf{Q}$ is updated as follows:

$$
\mathbf{Q}^{t+1}=\mathbf{Q}^{t}+\gamma^{t}\left(\mathbb{B} \mathbf{Q}^{t}-\mathbf{Q}^{t}\right)
$$

Proposition 1 and (16)-(17) imply that $f\left(\mathbf{Q}^{t+1}\right) \geq f\left(\mathbf{Q}^{t}\right)$ for all $t$. The algorithm is summarized in Algorithm 1 and its convergence properties are given in the following theorem.

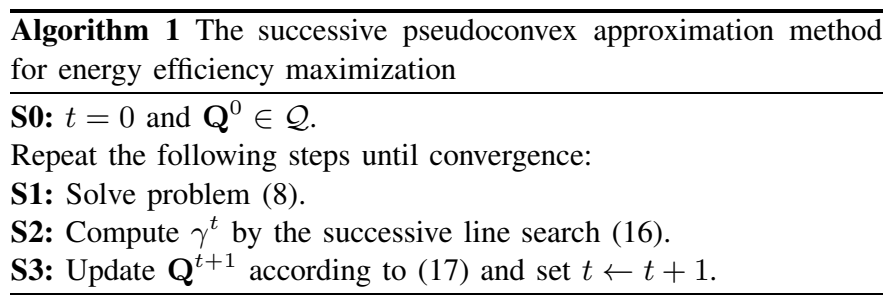

Theorem 2 (Convergence to a KKT point). Given a feasible initial point $\mathbf{Q}^{0} \in \mathcal{Q}$, the sequence $\left\{\mathbf{Q}^{t}\right\}$ generated by Algorithm 1 has a limit point, and every limit point is a KKT point of problem (3).

Proof: Although the constraint set $\mathcal{Q}$ of problem (3) is nonconvex, the sequence $\left\{\mathbf{Q}^{t}\right\}$ generated by Algorithm 1 is always feasible. To see this, we check if $\mathbf{Q}^{t+1}$ satisfies the QoS constraint $r_{k}\left(\mathbf{Q}^{t+1}\right) \geq R_{k}$ :

$$
\begin{aligned}
r_{k}\left(\mathbf{Q}^{t+1}\right) & =r_{k}\left(\mathbf{Q}^{t}+\gamma\left(\mathbb{B} \mathbf{Q}^{t}-\mathbf{Q}^{t}\right)\right) \\
& \geq \underline{r}_{k}\left(\mathbf{Q}^{t}+\gamma\left(\mathbb{B} \mathbf{Q}^{t}-\mathbf{Q}^{t}\right) ; \mathbf{Q}^{t}\right) \\
& \geq(1-\gamma) \underline{r}_{k}\left(\mathbf{Q}^{t} ; \mathbf{Q}^{t}\right)+\gamma \underline{r}_{k}\left(\mathbb{B} \mathbf{Q}^{t} ; \mathbf{Q}^{t}\right) \\
& \geq(1-\gamma) r_{k}\left(\mathbf{Q}^{t}\right)+\gamma R_{k},
\end{aligned}
$$

where the first inequality follows from the fact that $\underline{r}_{k}\left(\mathbf{Q} ; \mathbf{Q}^{t}\right)$ is a global lower bound of $r_{k}(\mathbf{Q})$, cf. (6), the second inequality from the concavity of $\underline{r}_{k}\left(\mathbf{Q} ; \mathbf{Q}^{t}\right)$, and the third inequality from the feasibility of $\mathbb{B} \mathbf{Q}^{t}$, i.e., $\mathbb{B} \mathbf{Q}^{t} \in \tilde{\mathcal{Q}}\left(\mathbf{Q}^{t}\right)$. Therefore $r_{k}\left(\mathbf{Q}^{t+1}\right) \geq R_{k}$ as long as $r_{k}\left(\mathbf{Q}^{t}\right) \geq R_{k}$. Since $\mathbf{Q}^{0}$ is feasible, $\mathbf{Q}^{t+1}$ is feasible by induction.

Since the constraint set $\mathcal{Q}$ is closed and bounded, the sequence $\left\{\mathbf{Q}^{t}\right\}_{t}$ is bounded and thus has a limit point. The proof for the latter argument follows the same line of analysis as [6, Theorem 2].

In the following, we discuss some properties and implementation aspects of the proposed Algorithm 1.

The approximate function in (4) is constructed in the same spirit as $[6,11]$ by keeping as much concavity as possible, namely, $r_{k}\left(\mathbf{Q}_{k}, \mathbf{Q}_{-k}\right)$ in $\mathbf{Q}_{k}$ and $P_{c}+\sum_{j=1}^{K} \operatorname{tr}\left(\mathbf{Q}_{k}\right)$ in $\mathbf{Q}$, and linearizing the nonconcave functions only, namely, $\sum_{j \neq k} r_{j}(\mathbf{Q})$. Besides this, the division operator is also kept. Therefore, the proposed algorithm is of a best-response nature and expected to exhibit a fast convergence behavior, as we shall later illustrate in numerical examples.

The function $\tilde{f}\left(\mathbf{Q} ; \mathbf{Q}^{t}\right)$ is pseudoconcave and the constraint set $\mathcal{Q}\left(\mathbf{Q}^{t}\right)$ is convex. Therefore the approximate problem (8) is pseudoconvex and can be solved in polynomial time by the interior point method proposed in [9]. The proposed algorithm could be implemented by a central unit which has the knowledge of the channel state information of the direct-link and cross-link channels, namely,
$\left(\mathbf{H}_{k j}\right)_{j, k}$. In practical networks, this central unit could be embedded in the centralized radio access network (CRAN). Please refer to [6] for more discussion on the implementation aspect.

A feasible initial point $\mathbf{Q}^{0}$ can be found by the penalty method, where the nonconvex inequality constraints are relaxed into the objective function through a penalty function, for example, the logarithmic and the inverse function. Interested readers are referred to [13] for a comprehensive review.

\section{The Proposed Parallel Successive Pseudoconvex APPROXIMATION ALGORITHM}

The approximate problem (8) in Step 1 of Algorithm 1 is not suitable for parallel implementation because the QoS constraint function $r_{k}^{+}(\mathbf{Q})-\bar{r}_{k}^{-}\left(\mathbf{Q}_{-k} ; \mathbf{Q}^{t}\right)$ coupling all variables $\mathbf{Q}_{1}, \ldots, \mathbf{Q}_{K}$ does not have a separable structure, and the standard decomposition techniques such as primal/dual decomposition is thus not applicable. In this section, building on the successive pseudoconvex approximation framework developed in the previous section, we propose an iterative algorithm where the approximate problems are suitable for parallel implementation and thus easy to solve.

To start with, we introduce an auxiliary variable $\left(\mathbf{Y}_{k}\right)_{k=1}^{K}$ and rewrite problem (3) as follows:

$$
\begin{array}{cl}
\underset{\mathbf{Q}, \mathbf{Y}}{\operatorname{maximize}} & \frac{\sum_{k=1}^{K} r_{k}\left(\mathbf{Q}_{k}, \mathbf{Q}_{-k}\right)}{\sum_{k=1}^{K}\left(P_{0, k}+\rho_{k} \operatorname{tr}\left(\mathbf{Q}_{k}\right)\right)} \\
\text { subject to } & \mathbf{Q}_{k} \succeq \mathbf{0}, \operatorname{tr}\left(\mathbf{Q}_{k}\right) \leq P_{k}, \\
& \log \operatorname{det}\left(\sigma_{k}^{2} \mathbf{I}+\mathbf{Y}_{k}\right)-r_{k}^{-}\left(\mathbf{Q}_{-k}\right) \geq R_{k}, \\
& \mathbf{Y}_{k}=\sum_{j=1}^{K} \mathbf{H}_{k j} \mathbf{Q}_{j} \mathbf{H}_{k j}^{H}, \forall k .
\end{array}
$$

In iteration $t$ at point $\mathbf{Q}^{t}$, the approximate problem is:

$$
\begin{array}{cl}
\underset{\mathbf{Q}, \mathbf{Y}}{\operatorname{maximize}} & \frac{\sum_{k=1}^{K} \tilde{r}_{k}\left(\mathbf{Q}_{k} ; \mathbf{Q}^{t}\right)-c \sum_{k=1}^{K}\left\|\mathbf{Y}_{k}-\mathbf{Y}_{k}^{t}\right\|_{F}^{2}}{\sum_{k=1}^{K}\left(P_{0, k}+\rho_{k} \operatorname{tr}\left(\mathbf{Q}_{k}\right)\right)} \\
\text { subject to } & \mathbf{Q}_{k} \succeq \mathbf{0}, \operatorname{tr}\left(\mathbf{Q}_{k}\right) \leq P_{k}, \\
& \log \operatorname{det}\left(\sigma_{k}^{2} \mathbf{I}+\mathbf{Y}_{k}\right)-\bar{r}_{k}^{-}\left(\mathbf{Q}_{-k} ; \mathbf{Q}^{t}\right) \geq R_{k}, \\
& \mathbf{Y}_{k}=\sum_{j=1}^{K} \mathbf{H}_{k j} \mathbf{Q}_{j} \mathbf{H}_{k j}^{H}, k=1, \ldots, K,
\end{array}
$$

where $c \geq 0$ is a given constant and the quadratic regularization term is introduced for numerical stability. The solution of problem (19) is denoted as $\left(\mathbb{B} \mathbf{Q}^{t}, \mathbb{B} \mathbf{Y}^{t}\right)$.

On solving the approximate problem (19). We apply the Dinkelbach's algorithm to solve problem (19) iteratively. At iteration $\tau$ of Dinkelbach's algorithm, the following problem is solved for a given and fixed $\alpha^{t, \tau}\left(\alpha^{t, 0}\right.$ can be set to 0$)$ :

$$
\begin{array}{cl}
\underset{\mathbf{Q}, \mathbf{Y}}{\operatorname{maximize}} & \sum_{k=1}^{K}\left(\tilde{r}_{k}\left(\mathbf{Q}_{k} ; \mathbf{Q}^{t}\right)-c\left\|\mathbf{Y}_{k}-\mathbf{Y}_{k}^{t}\right\|_{F}^{2}\right) \\
& -\alpha^{t, \tau} \sum_{k=1}^{K}\left(P_{0, k}+\rho_{k} \operatorname{tr}\left(\mathbf{Q}_{k}\right)\right) \\
\text { subject to } & \mathbf{Q}_{k} \succeq \mathbf{0}, \operatorname{tr}\left(\mathbf{Q}_{k}\right) \leq P_{k}, \\
& \log \operatorname{det}\left(\sigma_{k}^{2} \mathbf{I}+\mathbf{Y}_{k}\right)-\bar{r}_{k}^{-}\left(\mathbf{Q}_{-k} ; \mathbf{Q}^{t}\right) \geq R_{k}, \\
& \mathbf{Y}_{k}=\sum_{j=1}^{K} \mathbf{H}_{k j} \mathbf{Q}_{j} \mathbf{H}_{k j}^{H}, k=1, \ldots, K .
\end{array}
$$

We denote the solution of problem (20) as $\left(\mathbf{Q}^{d}\left(\alpha^{t, \tau}\right), \mathbf{Y}^{d}\left(\alpha^{t, \tau}\right)\right.$ (where "d" in the superscript stands for "Dinkelbach"). Then $\alpha^{t, \tau}$ is updated as follows:

$$
\alpha^{t, \tau+1}=\frac{\sum_{k=1}^{K} \tilde{r}_{k}\left(\mathbf{Q}_{k}^{d}\left(\alpha^{t, \tau}\right) ; \mathbf{Q}^{t}\right)-c\left\|\mathbf{Y}_{k}^{d}\left(\alpha^{t, \tau}\right)-\mathbf{Y}_{k}^{t}\right\|_{F}^{2}}{\sum_{k=1}^{K}\left(P_{0, k}+\rho_{k} \operatorname{tr}\left(\mathbf{Q}^{d}\left(\alpha^{t, \tau}\right)\right)\right)} .
$$

It follows from the convergence properties of the Dinkelbach's algorithm (cf. [2]) that $\lim _{\tau \rightarrow \infty} \mathbf{Q}^{d}\left(\alpha^{t, \tau}\right)=\mathbb{B} \mathbf{Q}^{t}$ and $\lim _{\tau \rightarrow \infty} \mathbf{Y}^{d}\left(\alpha^{t, \tau}\right)=\mathbb{B} \mathbf{Y}^{t}$ at a superlinear convergence rate. 


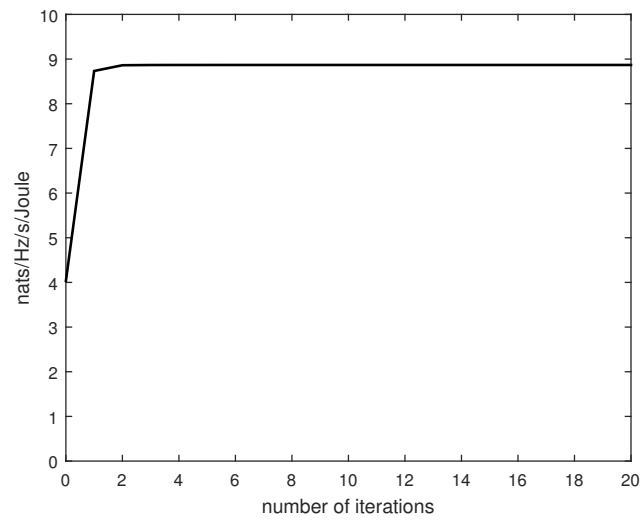

Figure 1. EE vs. number of iterations $t$

On solving problem (20). Problem (20) is convex and the coupling constraints have a separable structure, which can readily be exploited in the standard dual decomposition method. The Lagrangian of (20) is (after removing the constant term $\alpha^{t, \tau} \sum_{k=1}^{K} P_{0, k}$ ):

$$
\begin{aligned}
& L(\mathbf{Q}, \mathbf{Y}, \boldsymbol{\lambda}, \boldsymbol{\Sigma})= \\
& \quad \sum_{k=1}^{K}\left(\tilde{r}_{k}\left(\mathbf{Q}_{k} ; \mathbf{Q}^{t}\right)+c\left\|\mathbf{Y}_{k}-\mathbf{Y}_{k}^{t}\right\|_{F}^{2}-\alpha^{t, \tau} \rho_{k} \operatorname{tr}\left(\mathbf{Q}_{k}\right)\right) \\
& -\sum_{k=1}^{K}\left(\mathbf{\Sigma}_{k} \bullet\left(\mathbf{Y}_{k}-\sum_{j=1}^{K} \mathbf{H}_{k j} \mathbf{Q}_{j} \mathbf{H}_{k j}^{H}\right)\right) \\
& +\sum_{k=1}^{K} \lambda_{k}\left(\log \operatorname{det}\left(\sigma_{k}^{2} \mathbf{I}+\mathbf{Y}_{k}\right)-\bar{r}_{k}^{-}\left(\mathbf{Q}_{-k} ; \mathbf{Q}^{t}\right)-R_{k}\right),
\end{aligned}
$$

where $\lambda_{k}$ and $\boldsymbol{\Sigma}_{k}$ are the Lagrange multipliers associated to the constraints (20c)-(20d). The dual function $d(\boldsymbol{\lambda}, \boldsymbol{\Sigma})$ is

$$
d(\boldsymbol{\lambda}, \boldsymbol{\Sigma})=\max _{\left\{\mathbf{Q}_{k} \succeq \mathbf{0}, \operatorname{tr}\left(\mathbf{Q}_{k}\right) \leq P_{k}, \mathbf{Y}_{k} \succeq \mathbf{0}\right\}_{k=1}^{K}} L(\mathbf{Q}, \mathbf{Y}, \boldsymbol{\lambda}, \boldsymbol{\Sigma}),
$$

and the dual problem is

$$
\underset{\boldsymbol{\lambda} \geq \mathbf{0}, \boldsymbol{\Sigma}}{\operatorname{minimize}} d(\boldsymbol{\lambda}, \boldsymbol{\Sigma}) \text {. }
$$

Since the Lagrangian $L(\mathbf{Q}, \mathbf{Y}, \boldsymbol{\lambda}, \boldsymbol{\Sigma})$ is well decoupled across different variables for fixed dual variable $(\boldsymbol{\lambda}, \boldsymbol{\Sigma})$, the maximization problem in (23) can be decomposed into many smaller optimization problems that can be solved in parallel: for all $k=1, \ldots, K$,

$$
\begin{aligned}
& \mathbf{Q}_{k}^{L}\left(\lambda_{k}\right) \triangleq \\
& \underset{\mathbf{Q}_{k} \succeq \mathbf{0}, \operatorname{tr}\left(\mathbf{Q}_{k}\right) \leq P_{k}}{\arg \max } \tilde{r}_{k}\left(\mathbf{Q}_{k} ; \mathbf{Q}^{t}\right)-\lambda_{k} \mathbf{Q}_{k} \bullet\left(\sum_{j \neq k} \nabla_{\mathbf{Q}_{k}^{*}} r_{j}^{-}\left(\mathbf{Q}_{-j}^{t}\right)\right)
\end{aligned}
$$

and

$$
\mathbf{Y}^{L}\left(\boldsymbol{\Sigma}_{k}\right) \triangleq \underset{\mathbf{Y}_{k} \succeq \mathbf{0}}{\arg \max }\left\{\begin{array}{l}
\lambda_{k} \log \operatorname{det}\left(\sigma_{k}^{2} \mathbf{I}+\mathbf{Y}_{k}\right)-\boldsymbol{\Sigma}_{k} \bullet \mathbf{Y}_{k} \\
-c\left\|\mathbf{Y}_{k}-\mathbf{Y}_{k}^{t}\right\|_{F}^{2}
\end{array}\right\},
$$

where "L" in the superscript stands for "Lagrangian". Note that $\mathbf{Q}_{k}^{L}\left(\lambda_{k}\right)$ in (25) and $\mathbf{Y}^{L}\left(\boldsymbol{\Sigma}_{k}\right)$ in (26) are unique and have a closed form expression, cf. [14, Lem. 2] and [12, Lem. 7].

The dual problem (24) can be solved by the gradient projection algorithm and its gradient of $d(\boldsymbol{\lambda}, \boldsymbol{\Sigma})$ is

$$
\begin{aligned}
\nabla_{\lambda_{k}} d(\boldsymbol{\lambda}, \boldsymbol{\Sigma}) & =\log \operatorname{det}\left(\sigma_{k}^{2} \mathbf{I}+\mathbf{Y}_{k}^{L}\left(\boldsymbol{\Sigma}_{k}\right)\right)-\bar{r}_{k}^{-}\left(\mathbf{Q}_{-k}^{L}\left(\lambda_{k}\right)\right)-R_{k}, \\
\nabla_{\boldsymbol{\Sigma}^{*}} d(\boldsymbol{\lambda}, \boldsymbol{\Sigma}) & =\sum_{j=1}^{K} \mathbf{H}_{k j} \mathbf{Q}_{j}^{L}\left(\lambda_{k}\right) \mathbf{H}_{k j}^{H}-\mathbf{Y}_{k}^{L}\left(\boldsymbol{\Sigma}_{k}\right) .
\end{aligned}
$$

In iteration $v$ to solve problem (24), the dual variable is updated as follows:

$$
\begin{aligned}
\lambda_{k}^{t, \tau, v+1} & =\left[\lambda_{k}^{t, \tau, v}+\zeta^{t, \tau, v} \nabla_{\lambda_{k}} d\left(\boldsymbol{\lambda}^{t, \tau, v}, \boldsymbol{\Sigma}^{t, \tau, v}\right)\right]^{+}, \\
\boldsymbol{\Sigma}_{k}^{t, \tau, v+1} & =\boldsymbol{\Sigma}_{k}^{t, \tau, v}+\zeta^{t, \tau, v} \nabla_{\boldsymbol{\Sigma}^{*}} d\left(\boldsymbol{\lambda}^{t, \tau, v}, \boldsymbol{\Sigma}^{t, \tau, v}\right),
\end{aligned}
$$
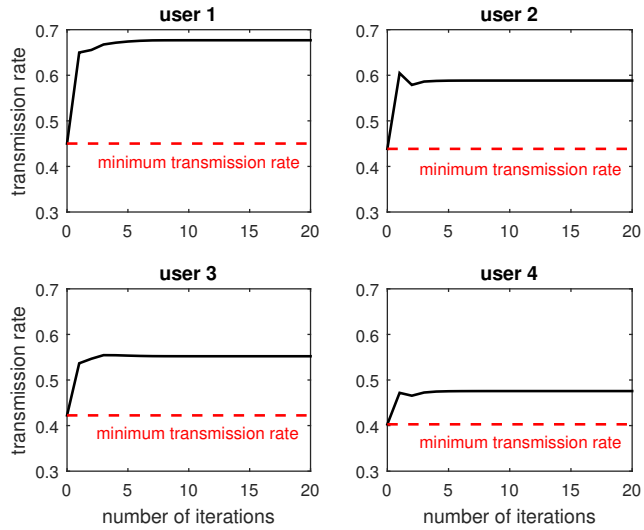

Figure 2. Individual transmission rate vs. number of iterations $t$

where $\boldsymbol{\lambda}^{t, \tau, 0}$ and $\boldsymbol{\Sigma}^{t, \tau, 0}$ can be set to $\mathbf{0}$. If the stepsizes $\left\{\zeta^{t, \tau, v}\right\}_{v}$ are properly selected, e.g., $\sum_{v} \zeta^{t, \tau, v}=\infty$ and $\sum_{v}\left(\zeta^{t, \tau, v}\right)^{2}<\infty$, then $\lim _{v \rightarrow \infty} \mathbf{Q}^{l}\left(\lambda_{k}^{t, \tau, v}\right)=\mathbf{Q}^{d}\left(\alpha^{t, \tau}\right)$ and $\lim _{v \rightarrow \infty} \mathbf{Y}^{l}\left(\lambda_{k}^{t, \tau, v}\right)=$ $\mathbf{Y}^{d}\left(\alpha^{t, \tau}\right)$.

The algorithm described above consists of three layers: the outer layer with index $t$, middle layer with index $\tau$, and inner layer with index $v$. The relationship of different layers is given as follows:

$$
\mathbf{Q}^{\star}=\lim _{t \rightarrow \infty} \lim _{\tau \rightarrow \infty} \lim _{v \rightarrow \infty} \mathbf{Q}^{l}\left(\lambda_{k}^{t, \tau, v}\right),
$$

where $\mathbf{Q}^{\star}$ is a KKT point of (3) and the limit with respect to $t$ is in the sense of subsequence convergence specified by Theorem 2 . Note that Although the proposed algorithm consists of three layers, its convergence speed is not negatively affected, because all updates have closed-form expressions and both the middle and inner layers converge very fast. Typically convergence is observed after a few iterations.

\section{Simulations}

We consider a cluster of $K=4$ cells with an inter-cell distance of $500 \mathrm{~m}$. The number of transmit antennas at the BS is $M_{T, k}=4$ and the number of receive antennas at the user is $M_{R, k}=4$. The power consumption at the zero RF output is $P_{0, k}=16 \mathrm{~W}$, the power budget normalized by the number of transmit antennas is $36 \mathrm{dBm}$, i.e., $P_{k} / M_{T, k}=36 \mathrm{dBm}$, and the slope of power consumption $\rho$ is 2.6; these parameters are mainly adopted from [10]. For each realization, all $K$ users are randomly located in the multi-cell space where each user falls into the respective hexagonal cell, and the simulation results are averaged over 100 realizations.

In Figure 1, we show numerically the convergence behavior of Algorithm 1. As the proposed algorithm is essentially an iterative ascent direction method, the objective value, namely, the sum EE, is monotonically increasing. It is easy to see that the achieved EE is quickly increased in the first 3 iterations, and a solution with a high accuracy is achieved after 5 iterations. From Figure 2 where the individual transmission rate achieved in each iteration is plotted, we can see that the QoS constraints are satisfied all the time.

\section{CONCLUSIONS}

In this paper, we have proposed an iterative algorithm based on the successive pseudoconvex approximation framework for the EE maximization problem in MIMO IC where all users have a guaranteed minimum transmission rate. The proposed algorithm is of a bestresponse type and exhibits guaranteed and fast convergence to a KKT point. The proposed algorithm is also suitable for parallel implementation based on dual decomposition, where each step has a closed-form expression. 


\section{REFERENCES}

[1] Z.-Q. Luo and S. Zhang, "Dynamic Spectrum Management: Complexity and Duality," IEEE J. Sel. Topics Signal Process., vol. 2, no. 1, pp. 57-73, Feb. 2008

[2] A. Zappone, L. Sanguinetti, G. Bacci, E. Jorswieck, and M. Debbah, "Energy-Efficient Power Control: A Look at 5G Wireless Technologies," IEEE Trans. Signal Process., vol. 64, no. 7, pp. 1668-1683, April 2016.

[3] O. Tervo, L. N. Tran, and M. Juntti, "Optimal energy-efficient transmit beamforming for multi-user miso downlink," IEEE Transactions on Signal Processing, vol. 63, no. 20, pp. 5574-5588, Oct 2015.

[4] S. Buzzi, C. L. I, T. E. Klein, H. V. Poor, C. Yang, and A. Zappone, "A survey of energy-efficient techniques for $5 \mathrm{~g}$ networks and challenges ahead," IEEE Journal on Selected Areas in Communications, vol. 34 , no. 4, pp. 697-709, April 2016.

[5] C. Pan, W. Xu, J. Wang, H. Ren, W. Zhang, N. Huang, and M. Chen, "Pricing-based distributed energy-efficient beamforming for miso interference channels," IEEE Journal on Selected Areas in Communications, vol. 34, no. 4, pp. 710-722, April 2016.

[6] Y. Yang and M. Pesavento, "A unified successive pseudoconvex approximation framework," IEEE Transactions on Signal Processing, vol. 65 , no. 13, pp. 3313 - 3328, Jul. 2017.

[7] A. Zappone, E. Bjornson, L. Sanguinetti, and E. Jorswieck, "Globally optimal energy-efficient power control and receiver design in wireless networks," IEEE Transactions on Signal Processing, vol. 65, no. 11, pp. 2844-2859, Jun. 2017.

[8] O. L. Mangasarian, Nonlinear programming. McGraw-Hill, 1969

[9] R. W. Freund and F. Jarre, "An interior-point method for fractional programs with convex constraints," Mathematical Programming, vol. 67, no. 1 , pp. 407-440, 1994.

[10] "Energy efficiency analysis of the reference systems, areas of improvements and target breakdown," INFSO-ICT-247733 EARTH D2.3. [Online]. Available: https://bscw.ict-earth.eu/pub/bscw. cgi/d71252/EARTH_WP2_D2.3_v2.pdf

[11] G. Scutari, F. Facchinei, P. Song, D. P. Palomar, and J.-S. Pang, "Decomposition by Partial Linearization: Parallel Optimization of Multi-Agent Systems," IEEE Trans. Signal Process., vol. 62, no. 3, pp. 641-656, Feb. 2014

[12] G. Scutari, F. Facchinei, L. Lampariello, S. Sardellitti, and P. Song, "Parallel and Distributed Methods for Constrained Nonconvex OptimizationPart II: Applications," IEEE Trans. Signal Process., vol. 65, no. 8, pp. 1945-1960, April 2017.

[13] D. P. Bertsekas, Nonlinear programming. Athena Scientific, 1999.

[14] Y. Yang, G. Scutari, D. P. Palomar, and M. Pesavento, "A parallel decomposition method for nonconvex stochastic multi-agent optimization problems," IEEE Trans. Signal Process., vol. 64, no. 11, pp. 2949-2964, June 2016 